\title{
Fourier Ptychography for lithography high NA systems
}

\author{
Atoosa Dejkameh $^{\mathrm{a}}$, Andreas Erdmann ${ }^{\mathrm{b}, \mathrm{c}}$, Peter Evanschitzky $^{\mathrm{b}}$, and Yasin Ekinci ${ }^{\mathrm{a}}$

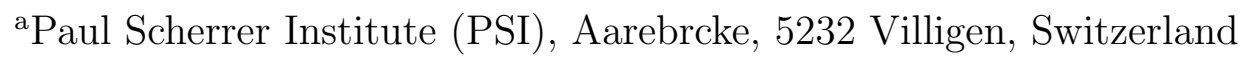 \\ ${ }^{\mathrm{b}}$ Fraunhofer Institute for Integrated Systems and Device Technology (IISB), D-91058 \\ Erlangen, Germany \\ ${ }^{c}$ Erlangen School for Master of Master of Advanced Optical Technology (MAOT), FAU \\ Erlangen Nurnberg, Paul-Gordan-str. 691052 Erlangen, Germany
}

\begin{abstract}
The aim of this research is to explore the limits of the basic Ptychography algorithm (FPA) at deep ultra violet (DUV) wavelength of 193 nanometers and for binary and phase shift masks. Furthermore, imaging at high numerical apertures involves polarization effects, which are not covered in the scalar phase retrieval algorithms of FPA. The impact of these effects on FPA is investigated for a test chart with feature sizes close to the resolution limit. The quality of the images before and after applying FPA was measured using different error criteria.

The Normalized Image Log Slope (NILS) is the criterion which is most sensitive to the lithographically important change in the edge sharpness of features. The Michelson contrast provides a global assesment of the image contrast. The Mean squared Error (MSE) provides an overall assessment of the image quality with respect to a known object. When FPA is used to recover high resolution images of a phase shift mask, it is found out that the edge sharpness is increased but the overall contrast is declined. Additionally, the printability of side lobes contributed to increase of MSE. After using a rigorous method to compute the mask diffraction spectrum instead of the conventional Fourier transform imaging, it is confirmed that thin object assumption is not at all accurate for high numerical aperture DUV imaging applications. For the first time, the polarization effects at large NAs are introduced to FPA and the output is evaluated. Here, we verified that polarization can be used to increase the edge sharpness at a specific direction.
\end{abstract}

Keywords: Fourier Ptychography, Polarization, High NA, Rigorous mask diffraction computation, DUV lithography, Phase shift mask

\section{INTRODUCTION}

Fourier Ptychography algorithm (FPA) is an imaging technique which combines computational methods with conventional imaging to surpass the physical limitation of the imaging system. By incorporating a LED matrix into the imaging setup, a set of intensity images with different illumination directions is collected. The acquired low resolution images are afterwards stitched together in frequency domain to form a larger (synthetic) diffraction spectrum. Consequently, a high resolution image with wide field of view ${ }^{1,2}$ is obtained. While variety of research is available for imaging systems with numerical aperture (NA) below 0.3 , this range of NA is not common for the state of the art lithographic imaging system which have higher NA, smaller wavelength and smaller feature sizes.

In this paper, we investigate the performance of FPA for typical DUV lithographic settings and for two types of lithographic masks, binary and phase shift mask (PSM). The binary mask has transmission values of zeros and ones. There is no phase change between feature and the background. There are of two types of PSM: strong phase shift mask and attenuated phase shift mask. As the attenuated phase shift mask is beneficial for imaging of semi-dense features, we adopt this method through out this research. The attenuated PSM has a 180 degrees phase shift between the background and the features and the transmission value of features is a value between zero and one, commonly between 6 and 20 percents. The efficiency of the diffraction orders is dependent on this transmission value. Considering the background transmission to be one, as the transmission value of the 180 degrees phase shifted feature increases, we witness a drop in the efficiency of zeroth order while the first order gains efficiency. This phase difference makes the edges steeper due to destructive interference at the vicinity of

Computational Optics II, edited by Daniel G. Smith, Frank Wyrowski, Andreas Erdmann, Proc. of SPIE Vol. 10694, 106940B - (C) 2018 SPIE · CCC code: 0277-786X/18/\$18 · doi: 10.1117/12.2311332 
the mask edges. Therefore, we expect a better image quality when imaging a phase shift mask. The draw back of the attenuated PSMs is the risk of printing the side lobes due to side lobes of image features.

Imaging of small features at high numerical apertures involves polarization effects, which are not covered in the scalar phase retrieval algorithms such as FPA. Diffraction from small objects has to be described by rigorous numerical solutions of the Maxwell equations. In contrast to the thin masks or Kirchhoff approach, these rigorous models do not consider the shift invariance for different incidence angles ${ }^{3,4}$, a basic assumption of FPA. The impact of these effects on FPA recovered intensities is investigated for a resolution chart in section 3 . The quality of the images before and after employing FPA was measured using several criteria. In this research, we asses several Error criteria in order to find the one which can most accurately describe the FPA recovered image.

In the following, we explain the simulation setup parameters, followed by the evaluation of image quality based on the introduced error criteria. After a discussion of typical results and possible directions of future research are outlined.

\section{SIMULATION AND EVALUATION DESCRIPTION}

In this section, we first discuss the mask geometry. Afterwards, the parameters and methods of image simulation are explained.

\subsection{Mask characterization}

In this section we look into the characteristics of the resolution chart mask. The binary mask has zero transmission for the features and hundred percent transmission for the background. For PSM, we look into cases with feature transmission of 6 and 20 percent in a 100 percent transmission background, as they are the conventional cases in projection lithography community. The mask pattern is the same for both binary and PSM. The mask contains 20 regions; see Fig. 1. Each regions is consist of three lines, oriented in either along x-axis (horizontal) or y-axis (vertical). We intend to investigate the performance of FPA when applied to features comparable to the size of the resolution of the imaging system.

The feature size depends on the NA of the imaging system, the technology factor $k_{1}$ and the wavelength. The pitch size of the pattern, that is the distance between neighboured lines, is calculated using the resolution formula,

$$
\text { pitch }_{\min }=\frac{k_{1}}{\lambda}
$$

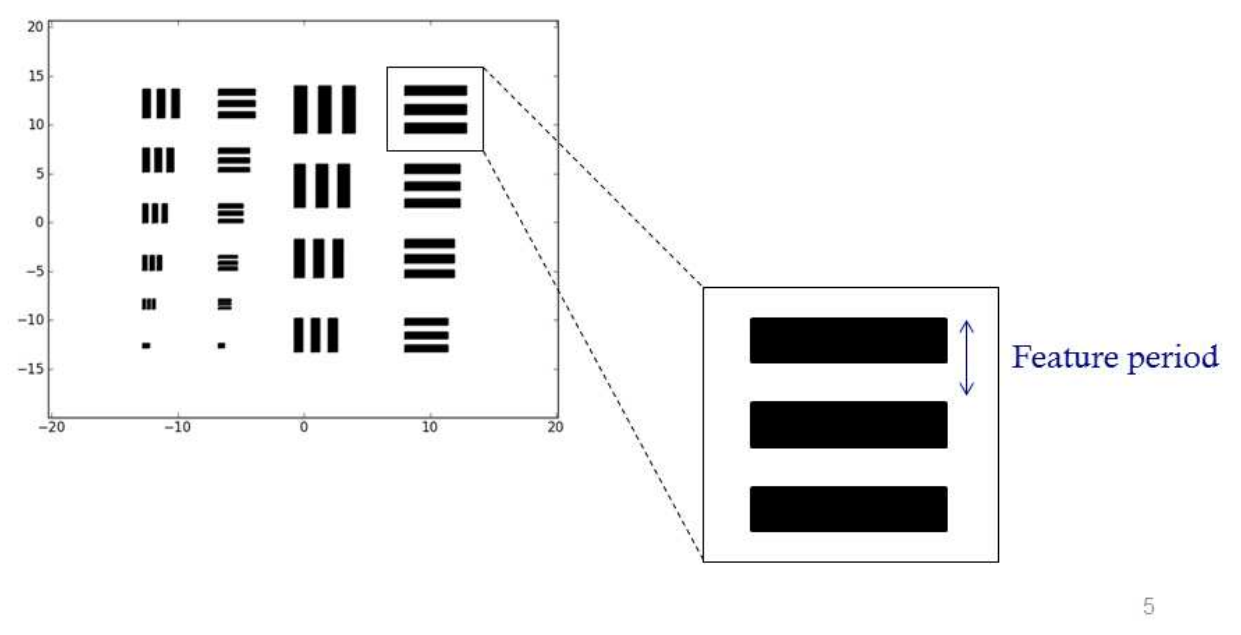

Figure 1. Resolution chart mask. 
The width of each line and the spacing between them are half of the feature period. The feature sizes are scaled by setting $k_{1}$ to ten values between 0.1 and 1 , in steps of 0.1 and multiplying it to $\frac{\lambda}{N A}$. Here, $k_{1}$ is a variable used as a step unit for changing pitch size. The largest feature has $k_{1}$ of one, at upper right. For example, for NA of 0.1 and wavelength of 193 nanometers, the pitch size is 1930 nanometers for the largest feature and the line width is 965 nanometers. The periodicity decreases in steps of 0.1 for other features, the lowest periodicity (smallest feature) has size of $0.1 \frac{\lambda}{N A}$, at lower left, See Fig. 1.

\subsection{Simulation}

When the size of features of the mask is comparable to the wavelength of the light, the method for the computation of light diffraction from the mask becomes increasingly important. The choice of the method of simulation of the diffraction field at the mask depends on the required accuracy and the computational power. We use two methods to describe the diffraction at the mask in this research: a thin mask (scalar diffraction theory) and a thick mask model (rigorous electromagnetic field simulation).

For a thin object, the diffraction spectrum is independent from the illumination angle. A change of the illumination direction shifts the diffraction spectrum according to the modification of the illumination direction, but leaves the magnitudes and phase values of the diffraction orders unnchanges. In other words, the diffraction spectrum is shift invariant. The mask has a negligible thickness and can be simulated as a complex two dimensional array. The complex array contains the transmission and the phase information of the mask. The mask diffraction spectrum is the Fourier transformation of the mask layout function. This so called scalar Kirchhoff mask, was widely used in low NA optical lithography. This method reduces the computation expenses while on the other hand, lowering the accuracy for thick/highly scattering object.

When the size of features on the mask is in the order of the wavelength, the effects of feature thickness become distinct. At this point this effects should be taken into account for calculating the mask diffraction spectrum. ${ }^{5}$ In this work we employ scalar diffraction theory, that is the conventional Fourier transforms, for the simulations of imaging systems with NA of 0.1. For NA of 0.9, we have employ the Waveguide method, an adaptation method of rigorous coupled wave algorithm (RCWA) for lithographic applications. The investigations in this research are computed using the built-in Waveguide method in Fraunhofer IISB lithography simulation software Dr.LiTHO. The basic of idea behind this approach is that the mask geometry is divided into parallel planes, perpendicular to the propagation direction. In order to reduce the three dimensional problem into a two-dimensional problems, the mask is decomposed into two dimensional layers with rectangles of certain refractive index and extinction coefficient. For each layer, the electromagnetic field is expanded into Fourier series up to a certain number of orders. The electromagnetic field distribution is then computed by solving the corresponding eigenvalue problem ${ }^{6}$

The lens is assumed to be aberration free with a sharp circular aperture. Therefore, the values of the pupil function is one inside NA and zero everywhere else. We consider two imaging systems. Initially, the imaging system has NA of 0.1, the mask is illuminated with a LED matrix constructing a synthetic NA of 0.4. The detector has pixels of size 128 nanometers and collects a 256 by 256 pixels area. The second system has NA of 0.9, this NA is later on expanded to 1.5. The detector pixel size is set to 21.4 nanometers. As mentioned before, the feature size on the mask is dependent on the NA, therefore the mask for the NA 0.9 system, has comprehensively smaller features.

\subsection{Evaluation}

One of objectives of this research was to explore the conventional image quality assessment methods in order to understand the sensitives to changes in the feature shape and its contrast to the background. The goal is to later use this error criteria as a judge for future optimization of the algorithm for lithographic imaging applications.

The error criteria used to evaluate the quality of the retrieved image are: normalized image log slope (NILS), Mean square error (MSE) and Michelson contrast (MC). We examined Structural similarity measure, mutual information and Weber contrast but they lacked the sensitivity required to asses lithography images. In addition, an important factor to consider is the initial quality of the low resolution alongside the recovered images. Figure 2, depicts the error measurement location for each of these methods. The NILS value is always measured at the two ends of the feature edges. 
Michelson contrast assumes the viewer is sensitive to the sum of the background and features intensity value, and therefore is suitable for measuring the contrast of line and spaces and patterns for which the background and foreground are not clearly defined,

$$
C_{\text {Michelson }}=\frac{I_{\max } / I_{\min }}{I_{\max }+I_{\min }}
$$

Where maximum intensity $I_{\max }$ and minimum intensity $I_{\min }$ indicate the background and feature intensity respectively. Michelson contrast is a flexible local measure as it doesn't rely on the intensity value at the exact edge location but on the other hand it doesn't disregard the expectation of the change in the intensity due to presence of a feature.

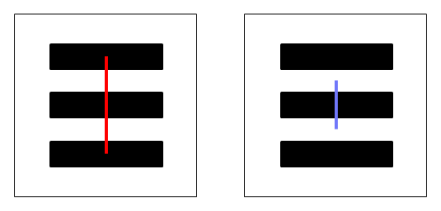

Figure 2. Measurement location: Michelson (red) and NILS (blue)

NILS is a very localized measure since it is exactly measured at the edge of the feature. NILS is used in lithography community as an indicator of printability but here we use it as an indicator of localized edge sharpness. The Width of the features is multiplied to the result since conventionally in lithography most of the variations in the edge of resist are expressed as percentage of nominal width.

$$
N I L S=w \frac{d(\ln I(x))}{d x}
$$

where $I(x)$ is the edge cross section intensity and $\mathrm{x}$ is the spatial coordinate of the edge. It is divided by I to remove the intensity dependency. The logarithmic scale provides a better demonstration of the sensitivity of the detector, namely the photo resist.

Mean square error (MSE) is the most conventional error measure in the scientific community ${ }^{7,8}$. The ease of implementation and its wide spread are the reason behind its popularity. MSE is a global error measurement as it gives the same truth weightage to both the features and the background.

$$
M S E=\frac{1}{M N} \sum_{i=0}^{N-1} \sum_{j=0}^{M-1}\left(I_{i j}-I_{r e f}\right)^{2}
$$

Where $I_{i j}$ are the measured and $I_{r e f}$ are the reference image values.

\section{RESULTS}

\subsection{NA 0.1: Binary and Phase Shift Masks}

High resolution images of a binary mask, a phase shift mask with 6 percent transmission and a phase shift mask with 20 percent transmission is reconstructed using FPA. Referencing to figure 3, there is no difference between resolution of reconstructed intensities. For all the cases, the synthetic NA of 0.4 improves the resolution up to the $k_{1}$ of 0.25 . In Figure 4, the sub-patterns from previous figure are separated and ordered with increasing $k_{1}$. In the upper section, the difference in the low resolution patterns is not visible. The interference effects are enhanced in recovered image, while the contrast drop for smaller $k_{1}$ features.

We obtain different performance evaluation based on different error criteria - see Figure 5 . The edge sharpness or NILS is improved while the MSE indicates a larger error after increasing the transmission of the mask feature and phase shifting them. We expect a sharper slope for the PSM due to interference effects which is validated in 


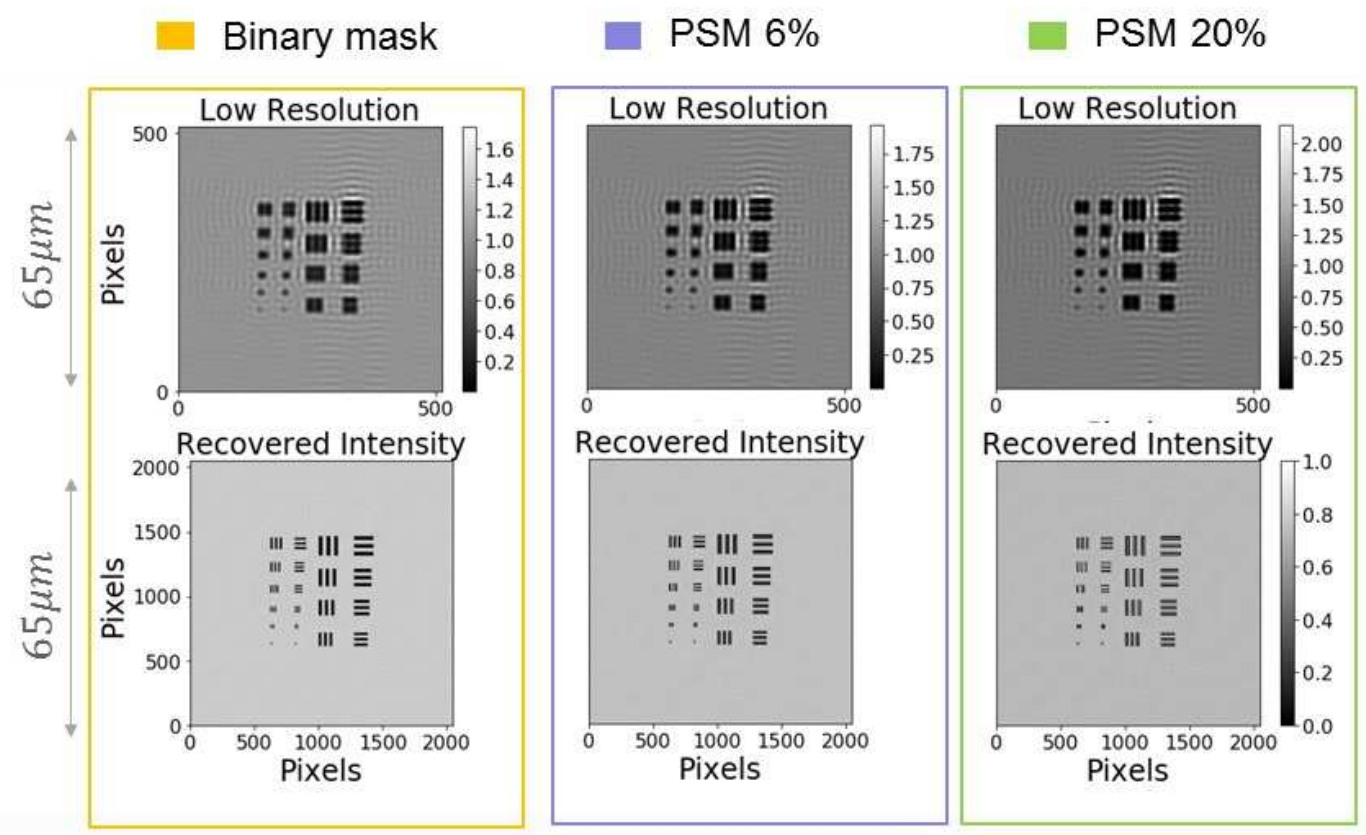

Figure 3. Comparison of low resolution(upper row) and recovered images(lower row) of Binary mask, PSM with feature transmission 6 percent and of PSM with feature transmission 20 percent.

fig. 5. The drops of NILS at $k_{1}$ of 0.6 to 0.8 is assumed to be due to incomplete presence of diffraction orders for those pitch sizes. But further analysis is required to confirm this hypothesis. MSE plots indicate a growth of the error value compare to binary mask. As MSE gives the same truth weightage to the feature and the background, it recognizes the side lobes and the interference effects as errors. The Michelson contrast plots vindicate MSE plot that for the extreme PSM case, the quality of the recovered image is not as good as the binary case. The contrast has drastically dropped even compared to the low resolution image.

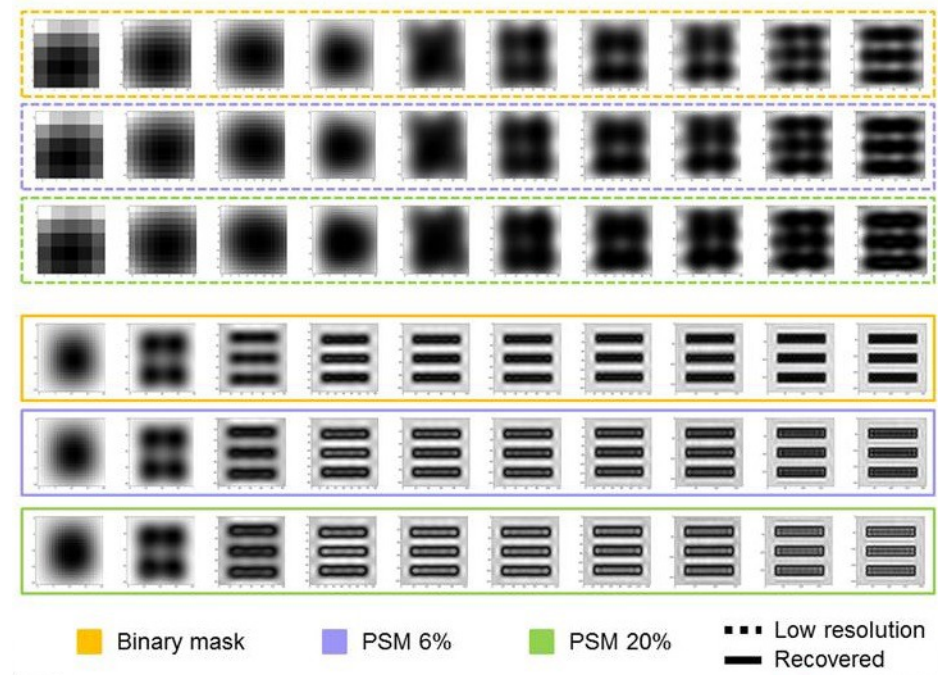

Figure 4. Comparison of low resolution and recovered features of PSM with varied transmission and $k_{1}-$ left to right. 

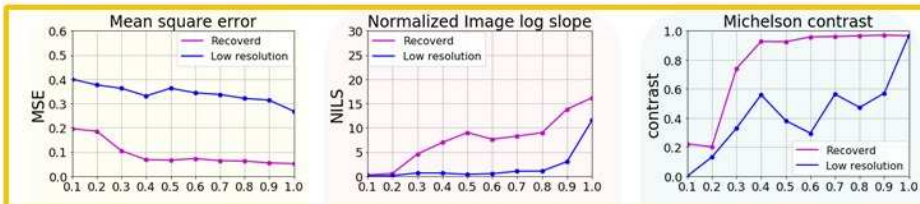

- Binary mask
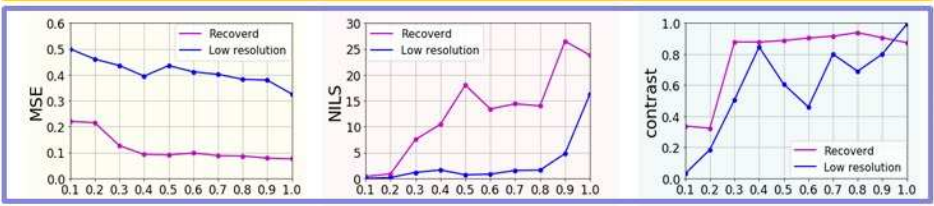

PSM 6\%
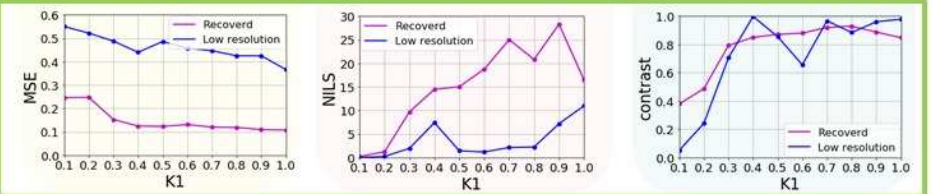

PSM $20 \%$

Figure 5. Comparison of MSE,NILS and Michelson contrast for Binary mask, PSM with feature transmission 6 percent and of PSM with feature transmission 20 percent. 


\subsection{NA 0.9 and polarization impact}

In the next part of the simulation, we vary the polarization of the light illuminating the sample. In Figure 6 , we can see a slight difference in direction of the interference effects for TE and TM polarized illumination. In the lower row, for the TE polarized illumination, the reconstructed intensity has better contrast for vertical lines and the opposite case in valid for TM polarized light.
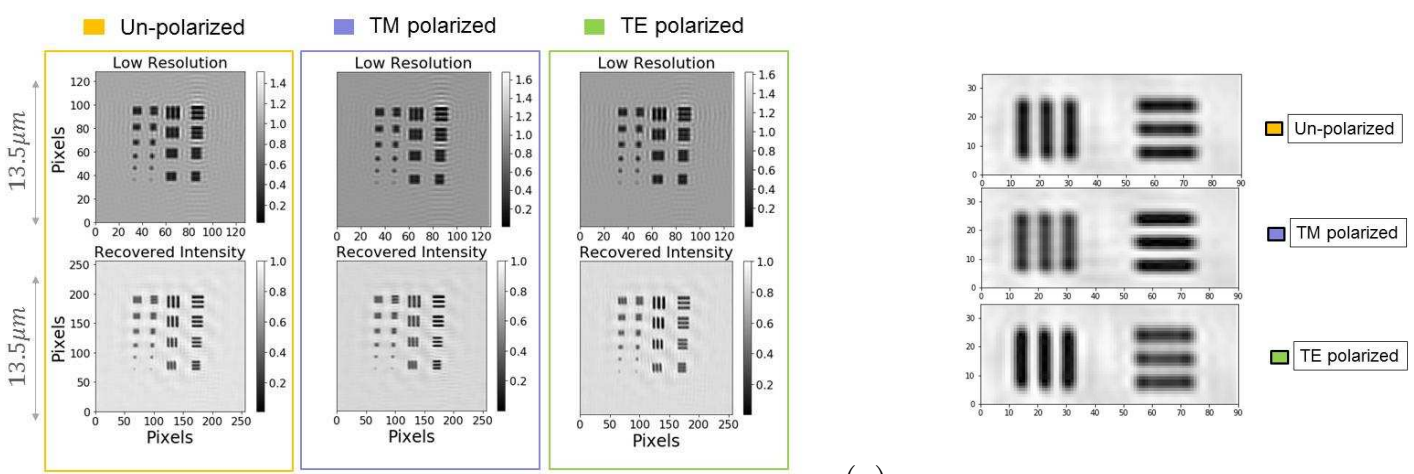

(a)

Figure 6. a:low resolution (upper row) and reconstruction (lower row) of unpolarized(left), TM polarized(center) and TE polarized(right). b: Zoom pattern corresponding to $k_{1}$ of 1

This phenomenon happens as the electrical component of light oscillates parallel to the line pattern.This effect is faintly present at low resolution images but it gets considerably magnified after applying FPA. Depicted in Figure 7, one can see that for unpolarized light the plots of NILS and Michelson contrast overlap. The slight difference is due to under sampling errors as for NA of 0.9 the mask features are very small. As anticipated, for TM polarized illumination both contrast and NILS are better in vertical direction. The opposite is true for TE polarized illumination. The improvement of feature edge slope is quantitatively more pronounced than the contrast change as the new NILS is almost twice as before.
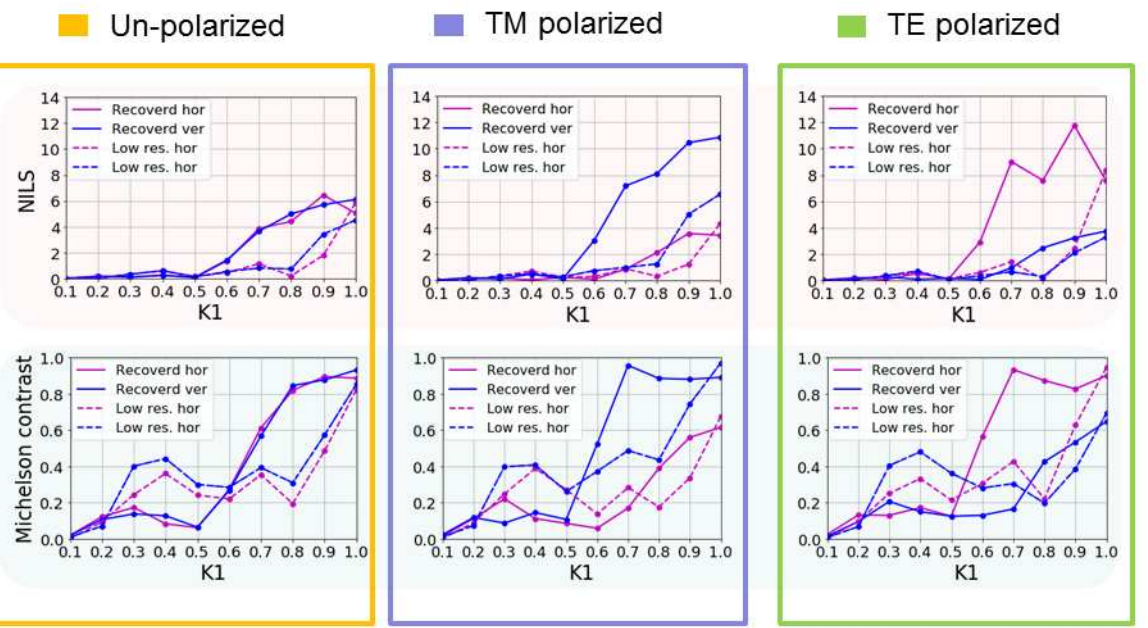

Figure 7. Error plots NILS(upper row) and Michelson contrast(lower row) for unpolarized, TM polarized and TE polarized light. 


\subsection{Performance of retrieval for rigorously simulated images}

Figure 8, demonstrates the strong impact of shadowing effects when the mask diffraction pattern and the images are rigorously computed. The rigorous model is NOT shift invariant and considers the effects of mask thickness. For the on-axis illumination the rigorous and the Kirchhoff simulation of light diffraction from the mask predict similar images, see lower right part of the figure. The difference between the computed images of the two models becomes prominent for the off-axis illumination. These differences are due to shadowing effects, which are neglected in the simplified Kirchhoff mode. The impact of these effects on the FPA reconstruction is visualized in Figure 9. By feeding the rigorously computed images to FPA, the retrieval quality declines by two orders of resolution. For the Kirchhoff model, up to $k_{1}$ of 0.6 is resolved while the resolved pattern in the rigorous model has $k_{1}$ of 0.8 .

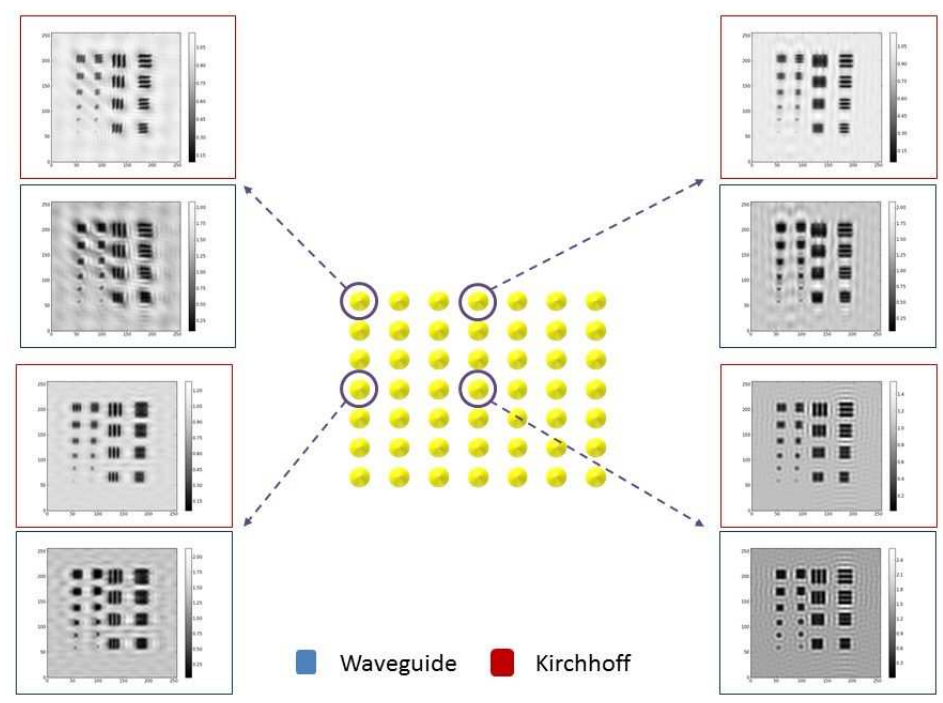

Figure 8. Waveguide and Kirchhoff computed low resolution images. Shadowing effects are visible for off axis illumination.
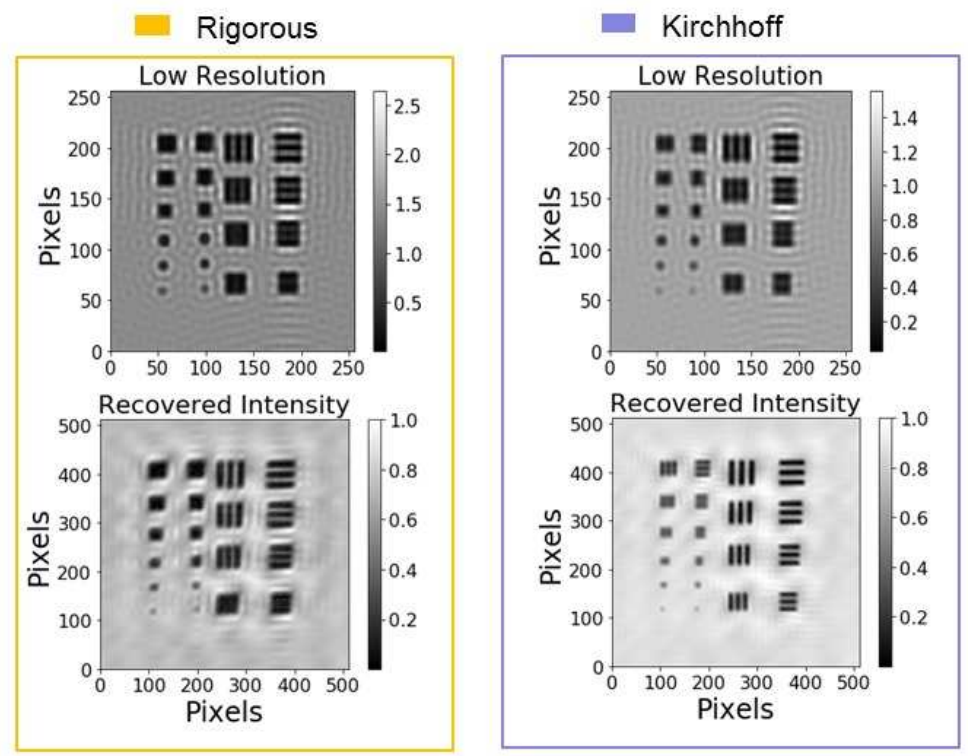

Figure 9. Comparison of reconstruction quality of waveguide and Kirchhoff image computation methods. 
Figure 10 is the plot of NILS and Michelson contrast, comparing the two mask diffraction computation approaches. The NILS plots are overlapping for Kirchhoff approach with minor differences due to lack of proper sampling. While the NILS plot of the rigorous approach is very volatile and the values of NILS or the two spatial directions do not match. The contrast values are not as sensitive to the direction of line pattern as much as the NILS values. The jump on the contrast values for the $k_{1}$ values between 0.3 to 0.7 can not be explained with the data values that we have.

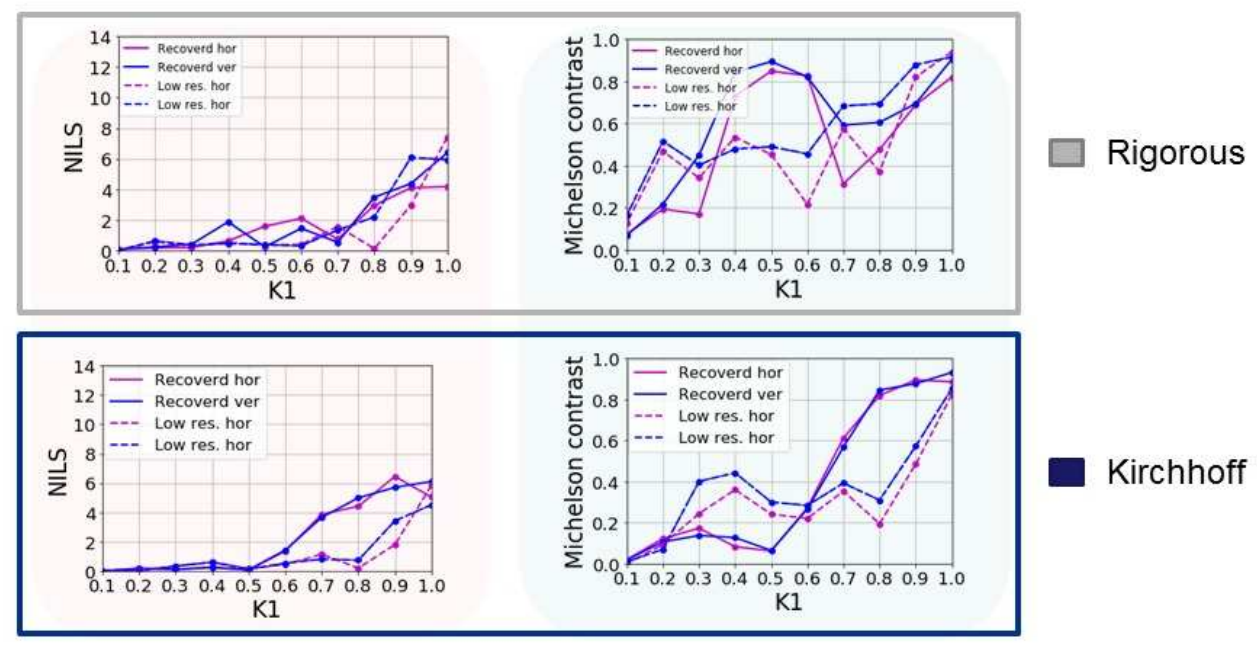

Figure 10. NILS and Michelson contrast error plots for Waveguide and Kirchhoff image computation methods.

\section{CONCLUSION}

Applying FPA to phase shifted masks amplifies the drop in the contrast while improving the normalized log slop. The MSE plot indicated an increased error. Here, we can see the importance of using the proper error criteria for evaluating algorithm performance. The MSE indicates a lower image quality while the NILS plot demonstrates higher edge sharpness. One can see that for different applications, there is a need for appropriate error criteria.

In the second part, we revealed that polarized illumination can be used alongside FPA to improve the resolution in the direction of oscillation of electric component of the electromagnetic field. Finally, we have shown the impact of mask thickness on the FPA performance. Two orders of magnitude are lost and the NILS and Michelson contrast show complicated unpredictable behavior.

\section{ACKNOWLEDGMENTS}

I would like to thank Dr. Ekinci for the intellectual and financial support he provided. I would also thank my colleagues at Fraunhofer and PSI for their feedbacks. My kind regards is to Prof. Roarke Horstmeyer for fruitful discussions and knowledge share.

\section{REFERENCES}

[1] G. Zheng, R. Horstmeyer, C. Y., "Wide-field, high resolution fourier ptychographic microscopy," J. Nat Photon 7, 739-7459 (2013).

[2] Zheng, G., [Fourier Ptychographic Imaging: A Matlab Tutorial], Morgan and Claypool Publishers (2016).

[3] A. Erdmann, F. Shao, V. A. T. F. P. E., "Modeling of mask diffraction and projection imaging for advanced optical and euv lithography," J. Modern Optics 58 (2010).

[4] J. Schermer, P. Evanschitzky, A. E., "Rigorous mask modeling beyond the hopkins approach," Proc. SPIE 6281 (2006). 
[5] Erdmann, A., "Mask modeling in the low k1 and ultrahigh na regime: phase and polarization effects (invited paper)," (2005).

[6] J. Schermer, P. Evanschitzky, A. E., "Rigorous mask modeling beyond the hopkins approach," (2006).

[7] Ghanem, S. A. M., "Mutual information and minimum mean-square error in multiuser Gaussian channels," IEEE MMTC E-Letters, Special issue on Latest advances on Wireless and Mobile Communications and Networking, 2015 (2 2015).

[8] Dong, S., Bian, Z., Shiradkar, R., and Zheng, G., "Sparsely sampled fourier ptychography," Opt. Express 22, 5455-5464 (3 2014). 